ISSN 0001-6012/2016/58/2/52-55 Acta Médica Costarricense, (C 2016 Colegio de Médicos y Cirujanos de Costa Rica

\title{
Revisión
}

\section{Enfermedad arterial periférica y ejercicio} (Peripheral arterial disease and exercise)

\author{
Gerardo Quirós-Meza, ${ }^{1}$ Johanna Salazar-Nassar ${ }^{2}$ y Jaqueline Castillo-Rivas ${ }^{3}$
}

\section{Resumen}

La enfermedad arterial periférica afecta 8.3\% de la población de 60 años o más en Costa Rica. Al asociarse a aterosclerosis sistémica, el diagnóstico de la enfermedad arterial periférica se asocia a un riesgo mayor de futuros eventos cardiovasculares (infarto de miocardio, infarto cerebral, muerte prematura). El síntoma clásico de la arteriopatía de los miembros inferiores es la claudicación intermitente: dolor en la pierna desencadenado por el esfuerzo que se resuelve con el reposo. Los sujetos con este síntoma presentan dificultad para caminar y disminución de la calidad de vida. El tratamiento médico óptimo (control de los factores de riesgo asociados, estilo de vida saludable, cilostazol 100mg dos veces al día), el tratamiento quirúrgico y el tratamiento endovascular son varias de las opciones terapéuticas para los pacientes con enfermedad arterial periférica sintomáticos. Actualmente, la opción más eficaz y a menor costo para mejorar la claudicación intermitente es el caminar. En esta revisión se presentan generalidades de Enfermedad Arterial periférica y los beneficios derivados del ejercicio en pacientes con claudicación intermitente con el propósito que el personal de salud tenga una guía integral de las ventajas asociadas al ejercicio en estos pacientes.

Descriptores: enfermedad arterial periférica, claudicación intermitente, ejercicio, caminar.

\section{Abstract}

Peripheral arterial disease affects $8.3 \%$ of the population with 60 years or older in Costa Rica.

Afiliación de los autores: 'Servicio de Vascular Periférico, Hospital "Dr. Rafael Ángel Calderón Guardia". ²Departamento de Neurociencias, Hospital "Dr. Rafael Ángel Calderón Guardia". 3Universidad de Costa Rica.

Abreviaturas: EAP, enfermedad arterial periférica; ES, ejercicio supervisado; ITB, índice tobillo/ brazo; MET, 1 MET es la cantidad de $\mathrm{O} 2$ consumida por kilogramo de peso corporal en un minuto por un individuo en reposo $y$ equivale a 3,5 mililitros $02 / \mathrm{kg} /$ min; ON, óxido nítrico; TMO, tratamiento médico óptimo 凶athos115@gmail.com
Associated with systemic atherosclerosis, patients with peripheral arterial disease have an increased risk of future cardiovascular events (myocardial infarction, cerebral infarction, premature death). The classic symptom of lower limb peripheral arterial disease is intermittent claudication: leg pain triggered by effort that resolves with rest. Subjects with intermittent claudication present difficulty to walk and a decreased quality of life. The optimum medical treatment (controlling associated risk factors, healthy life style, cilostazol 100mg two times a day), the surgical treatment and the endovascular treatment are all therapeutic options for symptomatic patients with peripheral arterial disease. At present, the most effective and low cost option to improve intermittent claudication is walking. In this article we present an overview of peripheral arterial disease and the benefits of exercise in patients with intermittent claudication, aiming to deliver a comprehensive guideline of the advantages associated to exercise in these patients the healthcare personnel.

Keywords: Peripheral arterial disease, intermittent claudication, exercise, walking.

Fecha recibido: 28 de agosto 2015 


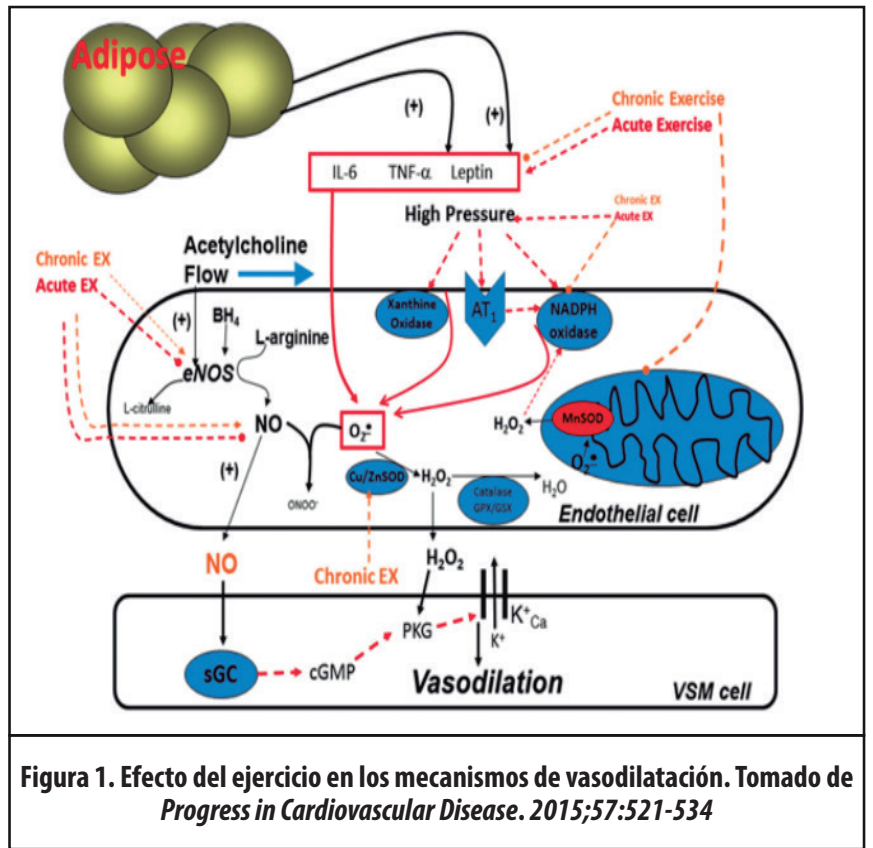

La enfermedad arterial periférica (EAP) afecta al 8,3\% de los costarricenses mayores de 60 años, con una tasa de mortalidad de 11 por cada 10000 habitantes. Estas cifras son semejantes a las de los países catalogados como desarrollados. ${ }^{1}$

La enfermedad arterial periférica es un indicador de aterosclerosis sistémica, por lo que se asocia a un riesgo aumentado de morbilidad y mortalidad cardiovascular. ${ }^{2}$ El síntoma clásico de la (EAP) es la claudicación intermitente (dolor en los grupos musculares distales, debido a una obstrucción arterial crónica, generalmente en miembros inferiores, que se desencadena por el ejercicio, desaparece en reposo y se reproduce al realizar el mismo esfuerzo físico). ${ }^{1-3}$ Estudios clínicos randomizados han demostrado que caminar mejora la calidad de vida de los sujetos claudicantes..$^{48}$ Conocer el impacto del ejercicio sobre la enfermedad arterial periférica constituye un gran paso para desarrollar programas de rehabilitación vascular y proponerlos como parte de políticas sanitarias dentro del sistema nacional de salud. ${ }^{9 \cdot 10}$

La enfermedad aterosclerótica, principal causa de enfermedad arterial periférica, es una patología sistémica inflamatoria crónica, fibroproliferativa, caracterizada por la existencia de placas ricas en lípidos, en la pared de las arterias de mediano y gran calibre. Este fenómeno provoca un engrosamiento y endurecimiento de la pared arterial, lo que reduce la luz vascular y limita la circulación sanguínea, con 0 sin manifestaciones clínicas. ${ }^{1-8}$

\begin{tabular}{|ll|}
\hline \multicolumn{2}{|c|}{ Cuadro 1. Clasificación de la isquemia crónica, } \\
según Fontaine ${ }^{17}$
\end{tabular}

La historia natural de la placa de ateroma comienza por la penetración de colesterol-LDL circulante en el subendotelio donde es oxidado. Las LDL-oxidadas atraen monocitos circulantes que se transforman en macrófagos. Estos monocitos / macrófagos al fagocitar por medio de receptores "basura" cantidades de LDL -oxidadas que no pueden destruir, se transforman en células espumosas. Secundariamente, estas se acumulan dentro de la íntima y forman estrías lipídicas que se reagrupan y constituyen el centro lipídico. Este es recubierto por una capa fibrosa constituida por la matriz extracelular y células musculares lisas provenientes de la media. La ruptura de la placa por metaloproteinasas deletéreas seguida por reparación, es el mecanismo por el cual el trombo intraplaca crece y reduce la perfusión distal. ${ }^{11-14}$

Estudios epidemiológicos sugieren que los pacientes con enfermedad arterial periférica aterosclerótica poseen una severa disfunción endotelial, y probablemente se deba a los efectos combinados del estrés oxidativo, disminución en la producción de óxido nítrico, aumento de la endotelina -1, incremento de sustancia proinflamatorias (Figura 1). ${ }^{13}$ Pellegrino y colaboradores demostraron que la disfunción endotelial en pacientes con EAP se correlaciona con la reserva del flujo coronario y con una elevada incidencia de eventos coronarios. ${ }^{15-18}$

La enfermedad arterial se diagnostica inicialmente con la historia clínica, la inspección y la palpación de pulsos. La técnica más utilizada a nivel mundial es el índice tobillo/brazo (ITB), método que compara presiones sistólicas ipsilaterales en reposo, tomadas a nivel del tobillo (numerador) y a nivel del brazo (denominador). El valor de referencia normal del ITB oscila entre 0,9 y 1,39. El compromiso arterial de la extremidad inferior produce una disminución de la presión arterial sistólica a nivel del tobillo, con respecto a la presión sistólica del brazo. ITB bajos, es decir, ITB $<0,9$, indican enfermedad arterial periférica. Cuanto más bajo el índice, la EAP es más severa y se correlaciona con mayor mortalidad cardiovascular. ${ }^{1-3}$ Hay dos clasificaciones para estratificar a los pacientes con isquemia crónica de la extremidad (Cuadros 1 y 2). ${ }^{17}$

En los pacientes con enfermedad arterial periférica, la isquemia crónica se asocia a disfunción endotelial (Cuadro 3).13,19

El objetivo terapéutico de la EAP es aumentar la distancia máxima de marcha, mejorar la capacidad funcional y disminuir el riesgo cardiovascular. ${ }^{20-25}$ El tratamiento puede ser médico,

\begin{tabular}{|ll|}
\hline \multicolumn{2}{|c|}{$\begin{array}{c}\text { Cuadro } \\
\text { 2. Clasificación de la isquemia crónica, } \\
\text { según Rutherford }{ }^{17}\end{array}$} \\
\hline Grado 0 & Asintomático \\
Grado 1 & Claudicación leve \\
Grado 2 & Claudicación moderada \\
Grado 3 & Claudicación severa \\
Grado 4 & Dolor isquémico en reposo \\
Grado 5 & Pérdida de tejidos menores \\
Grado 6 & Gangrena \\
\hline
\end{tabular}




\section{Cuadro 3. Consecuencias fisiológicas de la isquemia crónica de la extremidad}

Asociaciones determinadas

Número y tamaño de las fibras musculares tipo 1

Densidad capilar en el músculo de la pierna

Niveles de acetilcarnitina en sangre y músculo

Acúmulo de lactato durante el ejercicio

Extracción máxima de oxígeno

Función endotelial de los vasos sanguíneos: niveles de óxido nítrico disminuidos por acción de adipoquinas y citoquinas

proinflamatorias

Marcadores sistémicos de inflamación

Marcadores sistémicos de coagulación

Aumento de la presión arterial durante el ejercicio

Posibles asociaciones

Vasodilatación compensatoria de los vasos

sanguíneos de

la extremidad

Transducción del sistema neurovascular simpático

Sensibilidad del baroreflejo

Sensibilidad del quimioreflejo

Capacidad coronaria dilatadora

Interacción del sistema renina-angiotensinaaldosterona

endovascular, quirúrgico, prescripción de ejercicio, o una combinación de todos. ${ }^{13,20-25}$

En los pacientes con EPA es preciso un adecuado control de los factores de riesgo cardiovascular (no fumar, evitar la obesidad, control de la glicemia, del colesterol, de la presión arterial y práctica de estilo de vida saludable). Las estatinas y los antiagregantes plaquetarios son necesarios en la prevención secundaria de enfermedades cardiovasculares., ${ }^{1,3,13}$ El cilostazol (inhibidor de fosfodiesterasa) es el único medicamento aprobado por la FDA para tratar la claudicación: en un lapso de 4 semanas se observa mejoría en el paciente. La dosis recomendada es $100 \mathrm{mg}$ dos veces al día, si no hay contraindicación. La evidencia epidemiológica sugiere que muchos pacientes no presentan mejoría con solo tratamiento médico, ya que estas drogas son incapaces de aumentar el flujo sanguíneo en la extremidad afectada, a los niveles vistos con otras terapias. ${ }^{1,3,12,25} \mathrm{La}$ angioplastía, el stenting y el bypass, son técnicas que permiten la restauración del flujo sanguíneo. Al remover la obstrucción, el aumento del flujo sanguíneo mejora los síntomas.,3,12

Los beneficios de la práctica del ejercicio en pacientes con EAP son múltiples y entre ellos se puede resaltar su efecto

\section{Cuadro 4. Beneficios del ejercicio en pacientes con EPA}

Beneficios funcionales

Aumento de la distancia máxima de marcha

Aumento de la distancia de marcha libre de dolor

Mejoría en la calidad de vida

Beneficios fisiológicos

Disminución de la inflamación sistémica

Aumento de la función endotelial: el estrés sobre la pared endotelial producido por el flujo en cizalla inducido por el ejercicio, estimula la producción de óxido nítrico.

Aumento de la capacidad oxidativa del músculo esquelético y aumento máximo de la extracción de oxígeno

Disminuye la resistencia a la insulina

antiaterosclerótico, al optimizar la función endotelial (aumenta la producción de óxido nítrico -ON-, y disminuye la cantidad de especies reactivas de oxígeno), por lo que la prescripción adecuada de ejercicio puede ser una terapia potencial para prevenir y tratar las enfermedades cardiovasculares (Cuadro 4 y Figura 1). ${ }^{13,29-31}$

Actualmente, el mecanismo que produce la disminución de $\mathrm{ON}$ es tema de controversia y se han postulado varias teorías. Entre estas se pueden citar las siguientes: disminución de la enzima óxido nítrico sintasa endotelial, reducción del sustrato de $\mathrm{L}$ arginina / o de la tetrahidrobiopterina, modificación de la expresión y de la actividad funcional de la enzima óxido nítrico sintasa endotelial, barrido extracelular de ON por especies reactivas de oxígeno; aumento de la producción de vasoconstrictores derivados del endotelio (Figura 1). ${ }^{13}$

Un concepto clave relacionado con el efecto protector del ejercicio es la cantidad de energía consumida al efectuarlo. La forma de expresarla ha sido mediante el consumo de oxígeno (O2) de la masa corporal en la unidad de tiempo (equivalente a medir el trabajo de un motor a combustión, por la cantidad de gasolina consumida en un periodo). Este consumo ha sido expresado en unidades MET (1 MET es la cantidad de O2 consumida por kilogramo de peso corporal en un minuto por un individuo en reposo), y equivale a 3,5 mililitros $\mathrm{O} 2 / \mathrm{kg} / \mathrm{min}$. El MET permite clasificar las actividades físicas en diferentes rangos, que van desde muy leve (caminar despacio es $2 \mathrm{MET}$ ) hasta muy intenso (correr a $6 \mathrm{mph}$ es $10 \mathrm{MET}$ ). Los pacientes con EPA poseen un pico de ejecución de ejercicio de 4 a 5 MET, y es por eso que tienen limitaciones al realizar actividades de la vida diaria. ${ }^{13,30-32}$

Los estudios randomizadosensujetos conEAP son pocos, debido a los estrictos criterios de inclusión. ${ }^{5}$ Hay dos estudios que vale la pena tomar en cuenta, pues aportan grandes enseñanzas, aunque las muestras son pequeñas. El estudio CLEVER (Claudication: Exercice Versus Endoluminal Revascularization) compara en 111 pacientes claudicantes con enfermedad aortoiliaca, el tratamiento 


\section{Enfermedad arterial periférica y ejercicio / Quirós-Meza et al.}

médico óptimo (TMO), que consta de cilostazol 100mg dos veces al día, más programa de ejercicio no supervisado, combinación de TMO más marcha supervisada (una hora tres veces por semana, según protocolos establecidos), o TMO más colocación de stent. Se demostró que a los 6 meses, el grupo con marcha supervisada o ejercicio supervisado (ES) mejoró la distancia de claudicación, comparándolo con los pacientes sometidos a stent. No obstante, la calidad de vida sentida por el paciente fue mayor en el grupo stent, que en el de ejercicio supervisado. ${ }^{4,5,22}$ El segundo estudio randomizado en pacientes claudicantes secundarios a enfermedad arterial periférica femoropoplítea, fue publicado por Mazari et al. Se demostró a los 6 meses, mejoría en términos de calidad de vida, tanto en pacientes con ejercicio supervisado, como en pacientes con tratamiento endovascular. ${ }^{33}$

La enfermedad arterial periférica es una manifestación de la aterosclerosis sistémica, por lo que la morbilidad y mortalidad cardiovascular es elevada. El síntoma clave es la claudicación intermitente: dolor en la pierna desencadenado por el esfuerzo, que se resuelve con el reposo. El tratamiento médico óptimo, el tratamiento quirúrgico y el tratamiento endovascular, son varias de las opciones terapéuticas para los pacientes claudicantes. Las guías internacionales coinciden en que la marcha es el tratamiento más eficaz y menos costoso para mejorar la claudicación intermitente (programa de ejercicio supervisado por un periodo mínimo de 30-45minutos, por lo menos tres veces por semana, por un periodo mínimo de 12 semanas, preferiblemente 6 meses o más). Se necesitan más estudios para comprender y valorar la relación entre pacientes con EAP y ejercicio.

\section{Referencias}

1. Quirós Meza G, Salazar J, Castillo J. Atención de los pacientes con enfermedad arterial periférica en los hospitales de la Caja Costarricense de Seguro Social. Acta Méd. Costarric. 2011; 53: 182-187.

2. Quirós Meza G, Salazar J, Castillo J, Carrillo P. Prevalencia y factores de riesgo de enfermedad aterosclerótica sistémica. Acta Méd. Costarric. 2014; 56: 6-11.

3. Quirós Meza G, Salazar J, Castillo J, Miranda P. Prevalencia de enfermedad arterial periférica, enfermedad carotidea aterosclerótica y factores de riesgo asociados en una población adulta mayor cantón de Garabito, Puntarenas. Neuroeje. 2013;25.

4. Murphy T, Cutlip D, Regensteiner J, Mohler E. Six-Month Outcomes From the Claudication: Exercise Versus Endoluminal Revascularization (CLEVER) Study. Circulation. 2012; 125:130-139.

5. Parmener B, Dieberg G, Phipps G, Smart N. Exercise training for health-related quelity of life in peripheral artery disease: A systemic review and meta-analisis. Vascular Medicine. 2015; 20:30-40.

6. Aronow Wilbert. Treatement claudication due to peripheral arterial disease. Angiol. 2015; 3:1.

7. Reynolds M, Apruzzese P, Galper B, Murphy T. Cost-Effectiveness of supervised exercise, stenting, and optimal Medical Care for Claudication: results from the Claudication: exercise versus endoluminal revascularization (CLEVER) Trial. J Am Heart Assoc. 2014; 3:1-11.

8. Falk E. Pathogenesis of atherosclerosis. J Am Coll Cardiol. 2006; 47:7-12.

9. Gommans L, Fokkenrood H, van Dalen H, Scheltinga. Safety of supervised therapy in patients with intermittent claudication. Journal of vascular surgery. 2011; 61:512-518.

10. Fokkenrood H, Bendermarcher B, Lauret G, Willigendael EM. Supervised exercise therapy versus non-supervised exercise therapy for intermittent claudication. Cochrane Database Syst Rev. 2013; 8:1-51.
11. Singh RB, Mengi S, Xu YJ, Arneja AS. Pathogenesis of atherosclerosis: a multifactorial process. Exp Clin Cardiol. 2012; 7:40-53.

12. Anderson JL, Halperin JL, Albert NM, Bozkurt B, Brindis RG, et al. Management of patients with peripheral artery disease (compilation of 2005 and $2011 \mathrm{ACCF} / \mathrm{AHA}$ guideline recommendations). A report of the American College of Cardiology Foundation/American Heart Association Task Force on Practice Guidelines. Circulation. 2013; 127: 1425-1443.

13. Phillips S, Mahmoud A, Brown M, Haus J. Exercise Interventions and peripheral Arterial Function: Implications for Cardio-Metabolic Disease. Progress in Cardiovascular Disease. 2015;57:521-534

14. Aboyans V, Criqui MH, Abraham P, Allison MA. Measurement and interpretation of the ankle braquial index: a scientific statement from the American Heart Association. Circulation. 2012; 126:2890- 2909.

15. Fowke FG, Rudan I, Aboyans V, Denenberg JO. Comparison of global estimates of prevalence and risk factors for peripheral artery disease in 2000 and 2010: a systemic review and analysis. Lancet. 2013; 382:1329-1340.

16. Go AS, Mozaffarian D, Roger VL, Benjamin. EJ. Heart disease and stroke statistics -2013 update: a report from the American Heart Association. Circulation. 2012; 127:6-245.

17. Goodney P. Patient clinical evaluation. EN: Rutherford's Vascular Surgery. 8th edition Chicago: Saunders, 2014: 202-213.

18. Pellegrino T, Storto G, Filardi PP, Sorrentino AR. Relationship between braquial artery flow-mediated dilatation and coronary flow reserve in patients with peripheral artery disease. J Nucl Med. 2005; 46:1997-2002.

19. Brief M, Schiffrin EL. Treatment of arterial remodeling in essential hypertension. Curr Hypertens Rep. 2013; 15:3-9.

20. Mays R, Regensteiner J. Therapy for Peripheral Artery disease. Circulation. 2014; 130: 929-931.

21. Englund E, Langham M, Ratcliffe S, Fanning M. Multiparametric Assessment of vascular function in Peripheral Artery Disease. Circ Cardiovasc Imagin. 2015; 8:1-10

22. Abbott J. D. Lessons learned from recent randomized clinical trials for intermittent Claudication. Circ Cardiovasc Interv. 2012;5:139-141.

23. Hiatt W, Rogers $\mathrm{K}$, Brass Eric. In clinical trials, is the 6-minute Walk test a better functional test of interventions for Peripheral Artery Disease than Treadmill Walking Tests?. Circulation. 2014; 130:69-78.

24. Mays R, Hiatt W, Cassely I, Rogers K. Community-based walking exercice for peripheral artery disease: An exploratory pilot study. Vascular Medicine. 2015;1-9.

25. Parmenter BJ, Raymond J, Singh MA. The effect of exercice on fitness and performance-based tests of function in intermittent claudication: a systemic review. Sports Med. 2013; 43:513-524.

26. Brass EP. Intermitent claudication: new targets for the drug development. Drugs. 2013;73: 999-1014.

27. Brass EP, Cooper LT, Morgan RE, Hiatt WR. A phase II dose-ranging study of the phosphodiesterasa inhibidor K-134 in patients with peripheral artery disease and claudication. J Vasc Surg. 2012; 55:381-389.

28. Brass EP, Koster D, Hiatt WR, Amato A. A systemic review and metanalysis of propionyl- L-carnitine effects on exercice performance in patients with claudication. Vasc Med. 2013;18:3-12.

29. Ainsworth BE, Haskell WL, Whitt MC, Irwin ML. Compendium of physical activities: an update of activity codes and MET intensities. Med Sci Sports Exerc. 2000; 32:498-504.

30. Sallis J, Floyd M, Rodriguez D, Saelens B. Role of built environements in physical activity, obesity, and cardiovascular disease. Circulation. 2012; 125:729-737.

31. Farb M, Ganley-Leal L, Mott M. Arteriolar function in visceral adipose tissue is impaired in human obesity. Arterioscler function in visceral is impaired in human obesity. Arterioscler Thromb Vasc Biol. 2012; 32:467-473.

32. Nicolaï S, Viechtbauer W, Kruidenier L, MD, Candel M. Reliability of treadmill testing in peripheral arterial disease: A meta-regression analysis. J Vasc Surg. 2009; 50: 322-329.

33. Mazari FAK, Khan JA, Carradice D, Samuel N. Randomized clinical trial of percutaneous transluminal angioplasty, supervised exercise and combined treatment for intermittent claudication due to femoropopliteal arterial disease. British J Surg. 2012; 99:39-48. 